\title{
Minimally invasive congenital cardiac surgery with unsnared inferior vena cava
}

\author{
Alvise Guariento $^{1}$, Claudia Cattapan ${ }^{1}$, Francesco Bertelli ${ }^{1}$, and Vladimiro Vida ${ }^{1}$ \\ ${ }^{1}$ University of Padua
}

May 8, 2020

\begin{abstract}
Here we report two cases of residual atrial septal defect (ASD) with inferior extension towards the inferior vena cava (inferior sinus venosus type). In both cases, patients underwent surgical repair of their ASD in childhood. However, signs of persistent hemodynamic right ventricular overload were detected at the last follow-up and a residual ASD was demonstrated. Both underwent successful minimally invasive surgical closure of the ASD by combining a right postero-lateral mini-thoracotomy (sub-axillary approach) and total peripheral cardiopulmonary bypass, avoiding snaring of the inferior vena cava. This allowed an optimal visualization of the residual intra-cardiac shunt and its subsequent closure.
\end{abstract}

\section{Keywords:}

- Congenital heart disease

- Minimally invasive surgery

- Surgical strategies

\section{TEXT}

\section{Introduction}

Atrial septal defects (ASD) can be found in different sites of the interatrial septum depending on their embryological origin. Sinus venosus ASD (SV ASD) are interatrial defects morphologically adjacent to the outlet of the superior (SVC) or inferior vena cava (IVC) [1]. Surgical closure of this type of defects is sometimes challenging due to a difficult surgical exposure (e.g. extension into the inferior vena cava orifice) or misidentification of contiguous structures (e.g. an enlarged Eustachian valve). For this reason, the need for reoperation for a residual ASD is not a rare event [2].

Over the past decade, surgical correction of less severe congenital heart disease (CHD) such as ASD has been increasingly performed through minimally invasive less traumatic surgical approaches $[3,4]$. In particular, the advantage of total or partial peripheral cardiopulmonary bypass (CPB) includes the possibility of avoiding IVC snaring during CPB. This proved particularly important for the visualization of SV ASD with inferior extension towards the IVC orifice.

\section{Technique}

In the current study, we report the cases of two female patients who underwent surgical re-intervention for residual SV ASD after initial repair in childhood (at the age of 4 years for Patient 1 and 10 months for Patient 2). Both index procedures were uneventful, and patients were discharged in good clinical conditions with no suspicion of residual intra-cardiac defects.

At follow-up, patients were asymptomatic. However, persistent right ventricular dilation with a significant residual ASD located at the junction between the right atrium and IVC was demonstrated. Both patients 
were scheduled for elective surgery considering that a percutaneous approach was not deemed feasible.

At the time of surgery, at the age of 32 years for patient 1 and 20 years for Patient 2, after surgical cannulation of the femoral vessels and percutaneous cannulation of the internal jugular vein, a peripheral CPB was started. A right postero-lateral mini-thoracotomy (or sub-axillary approach) was performed through a $4 \mathrm{~cm}$ incision in the fourth intercostal space just below the corner of the right scapula. Right lung exclusion was achieved in order to gain better surgical exposure.

After isolation of the mediastinal structures, with the aid of induced ventricular fibrillation (IVF), a right atriotomy was performed. While the SVC was snared before opening the atrium, the IVC was left unsnared to allow optimal visualization of the residual intra-cardiac shunt (Figure 1, Video 1). The residual ASD was closed with a heterologous pericardial patch (Figure 2). Total CPB time was 98 minutes for Patient 1 and 63 minutes for Patient 2. IVF time was 75 minutes and 24 minutes, respectively. The ventricular fibrillation was converted to sinus rhythm by direct current cardioversion shock in both, and the left cavities were cautiously deaired.

Surgical results were carefully reviewed by 2D-transesophageal echocardiography and confirmed through a bubble test. The postoperative course was uneventful, and patients were discharged home on postoperative day 7 (Patient 1) and 6 (Patient 2).

\section{Comment}

About 4-11\% of ASD are SV ASD defects [1]. The surgical closure of this type of defect can be particularly prone to residual ASD, due to their location [2]. In these cases, a percutaneous closure of the residual defect is unfeasible, due to their particular position. Therefore, a surgical procedure is always deemed necessary.

Minimally invasive techniques adopted to treat simple CHD are constantly evolving in the current era. This allowed to minimize surgical trauma while maintaining functional results [3]. The introduction of peripheral cannulation for CPB has further minimized the surgical access and expanded the spectrum of CHD that can be addressed with these techniques [4].

In our patients, the combination of a minimally invasive right axillary thoracotomy with total peripheral CPB and IVF allowed us to obtain a successful surgical result with a reduction in surgical trauma (due to minimal mediastinal tissue dissection e.g. no need for aortic cross-clamping, unsnared IVC) and a good aesthetic result (particularly important in female patients). Furthermore, in cases when a reintervention is required (like the cases presented here), it allowed us to avoid a resternotomy thus reducing the potential sources of bleeding both at the re-entry of the thoracic cavity and during the dissection of the tissues.

The establishment of a CPB with central cannulation without snaring of the IVC has already been described in the literature for the treatment of CHD with potentially complicated venous drainage. This was mainly performed for extra-cardiac Fontan procedures, demonstrating great feasibility with adequate venous drainage and no reduction in flow or circulatory arrest [5].

In our cases, peripheral cannulation of the femoral vein, with the tip of the cannula held below the junction of the right atrium and an unsnared IVC, allowed us to perform an uncomplicated CPB (e.g. no air locks) and to obtain a complete visualization of the defect and a definitive closure. In fact, IVC snaring can sometimes cause potential edge distortion of an SV ASD, especially in case with very low extension, making it difficult to visualize it.

This technique has provided excellent results at our center and it is now considered the gold-standard practice also in all patients with a large ostium secundum type ASD with inferior extension towards the IVC or with an inferior SV ASD. From 2008 to 2019, 12 patients were treated at first operation with a RPLMT and unsnared IVC without intraoperative and long-term complications.

Although limited, our experience shows that the combination of a minimally invasive approach with partial or total peripheral cannulation and unsnaring of the IVC has proven to be safe and effective. This technique 
allows an optimal visualization of the intracardiac anatomy in cases where a proper identification of the edges of the ASD is required.

\section{REFERENCES}

[1] Geva T, Martins JD, Wald RM. Atrial septal defects. Lancet. 2014 May 31;383(9932):1921-32.

[2] Banka P, Bacha E, Powell AJ, Benavidez OJ, Geva T. Outcomes of inferior sinus venosus defect repair. J Thorac Cardiovasc Surg 2011;142:517-22.

[3] Vida VL, Padalino MA, Bhattarai A, Stellin G. Right posterior-lateral minithoracotomy access for treating congenital heart disease. Ann Thorac Surg 2011;92:2278-80.

[4] Vida VL, Tessari C, Putzu A, Tiberio I, Guariento A, Gallo M, et al. The peripheral cannulation technique in minimally invasive congenital cardiac surgery. Int J Artif Organs Wichtig Publishing Srl 2016;39:300-3.

[5] Corno AF, Horisberger J, David J, Segesser LK Von. Right atrial surgery with unsnared inferior vena cava. Eur J Cardio-thoracic Surg 2004;26:219-20.

\section{Figure legend}

Figure 1. Graphic representations (A) and intraoperative view (B) of a residual inferior SV ASD. The IVC was left unsnared to allow optimal visualization of the residual intra-cardiac shunt. IVC: inferior vena cava, SV ASD: sinus venosus atrial septal defect.

Figure 2. Graphic representations (A) and intraoperative view (B) of residual ASD closure with a heterologous pericardial patch. IVC: inferior vena cava, SV ASD: sinus venosus atrial septal defect.

\section{Video legend}

Video 1. Video showing the surgical closure of a residual inferior sinus venosus atrial septal defect by combining a right postero-lateral mini-thoracotomy and a total cardio-pulmonary bypass, avoiding snaring of the inferior vena cava.
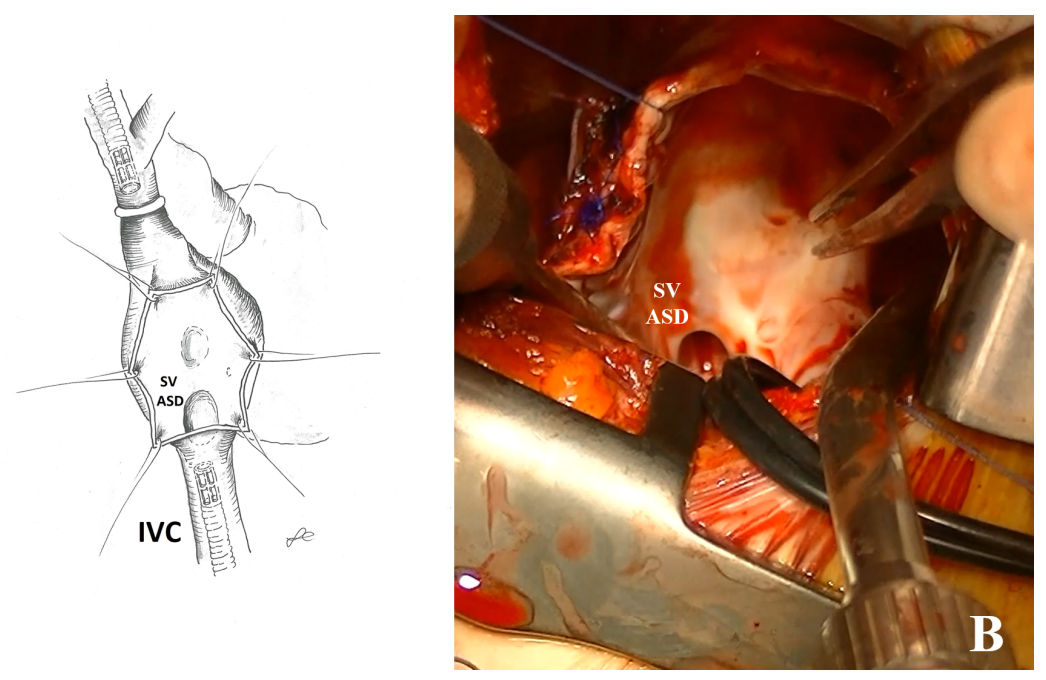


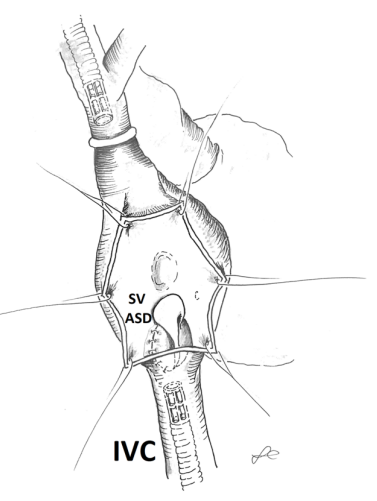

A

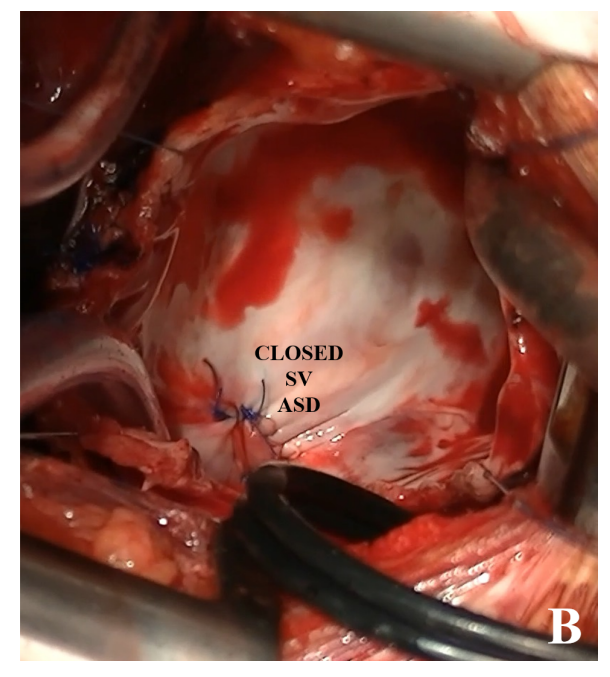

